\title{
Phosphorylation of STAT3 in Undifferentiated Pleomorphic Sarcoma Is Correlated with a Favorable Prognosis
}

\author{
Hirofumi Bekki ${ }^{a}$ Kenichi Kohashia ${ }^{a}$ Yuichi Yamada ${ }^{a}$ Kunio lura ${ }^{a}$ Takeaki Ishii $^{a}$ \\ Akira Maekawa $^{a}$ Hiroshi Otsuka $^{a}$ Hidetaka Yamamoto $^{a}$ Michiyuki Hakozaki $^{d}$ \\ Kazuki Nabeshimac ${ }^{c}$ Yukihide Iwamoto $^{\mathrm{b}}$ Yoshinao Oda ${ }^{a}$ \\ Departments of a Anatomic Pathology and ${ }^{b}$ Orthopedic Surgery, Graduate School of Medical Sciences, Kyushu \\ University, and ' Department of Pathology, Fukuoka University Hospital and School of Medicine, Fukuoka, and \\ ${ }^{\mathrm{d}}$ Department of Orthopaedic Surgery, School of Medicine, Fukushima Medical University School of Medicine, \\ Fukushima, Japan
}

\section{Key Words}

JAK-STAT pathway $\cdot$ SOCS3 $\cdot$ STAT3 $\cdot$ Undifferentiated pleomorphic sarcoma

\begin{abstract}
Objective: The Janus kinase (JAK)-signal transducer and activator of transcription (STAT) pathway plays a role in various biological processes. Phosphorylated STAT3 (p-STAT3) functions as a transcriptional factor, and suppressor of cytokine signaling 3 (SOCS3) is a potential inhibitor of STAT3. Here, we analyzed the status of the JAK-STAT pathway in undifferentiated pleomorphic sarcoma (UPS). Methods: We performed immunohistochemistry in 79 samples of UPS and Western blotting in 10 frozen samples. We also examined alterations in protein expression in the JAK-STAT pathway after the inhibition of phosphorylated Akt (p-Akt) or extracellular signal-regulated kinase ( $p$-Erk) in vitro. Results: Immunohistochemically, p-STAT3 and SOCS3 were positive in 59.7 and $55.8 \%$, respectively. Positivity for $\mathrm{p}$-STAT3 was significantly correlated with a better prognosis $(p=0.0006)$ and negatively with SOCS3 expression ( $p=0.0223$ ). Positivity for
\end{abstract}

\section{KARGER}

(C) 2016 S. Karger AG, Basel

E-Mail karger@karger.com

www.karger.com/pat
SOCS3 was significantly correlated with a worse prognosis $(p=0.0001)$. Western blotting analysis revealed that $p-S T A T 3$ expression was lower in tumor than in normal tissue. In vitro results demonstrated that there was no detectable change in the expression of $\mathrm{p}$-STAT3 regardless of the status of $\mathrm{p}-\mathrm{Akt}$ or p-Erk. Conclusion: p-STAT3 may be a useful prognostic factor for UPS.

(c) 2016 S. Karger AG, Basel

\section{Introduction}

Undifferentiated pleomorphic sarcoma (UPS) is a soft-tissue sarcoma that shows no line of differentiation [1]. Little is known about the activated proteins in UPS. Many cytokines exert their biological effects via the Janus kinase (JAK) signal transducer and activator of transcription (STAT) pathway [2]. Cytokines lead to the activation of receptor-associated JAK and JAK-mediated phosphorylation of the receptor cytoplasmic domains. STAT proteins are then phosphorylated, and they translocate to the nucleus and exert transcriptional control. Although sev-

Dr. Kenichi Kohashi

Department of Anatomic Pathology, Graduate School of Medical Sciences Kyushu University, Maidashi 3-1-1

Higashi-ku, Fukuoka 812-8582 (Japan)

E-Mail kohas@surgpath.med.kyushu-u.ac.jp 
eral studies have linked STAT3 to tumor growth and metastases in various types of neoplasms $[3,4]$, it was reported that STAT3 activation is also correlated with a better prognosis in some tumors $[5,6]$. STAT3 seems to have a double-sided effect on tumor progression. Some in vitro studies of the JAK-STAT pathway in malignant fibrous histiocytoma cell lines have been conducted $[7,8]$, but, to our knowledge, no study has analyzed the prognostic significance of the status of the JAK-STAT pathway in UPS.

The JAK-STAT pathway is influenced by signaling pathways such as the Akt/mammalian target of rapamycin (mTOR) pathway and the mitogen-activated protein kinase (MAPK) pathway. Akt is a serine/threonine kinase activated by phosphoinositide 3-kinase, and it activates downstream mTOR. The Akt/mTOR pathway plays diverse roles in normal oncogenic processes [9]. A study of osteoclasts showed that Akt activation is necessary for the expression of suppressor of cytokine signaling 3 (SOCS3), one of the potential inhibitors of STAT3 [10].

The MAPK pathway also affects the JAK-STAT pathway, as noted above. MAPK cascades play a key role in the transduction of extracellular signals to cellular responses. Extracellular signal-regulated kinase (Erk)1/2, one of the members of the MAPK cascade, antagonizes the phosphorylation of STAT3 [11].

We postulated that the phosphorylation of STAT3 would be inhibited by phosphorylated Akt (p-Akt) or Erk (p-Erk) because Akt/mTOR and MAPK pathways are activated in UPS [12]. Here, we analyzed the expression of STAT3 and SOCS3 in UPS and investigated whether the JAK-STAT pathway is influenced by Akt or Erk in vitro.

\section{Materials and Methods}

\section{Patients}

We studied 79 patients who were diagnosed with UPS (75 primary tumors, 3 recurrent tumors, and 1 metastatic tumor) and registered in the Department of Anatomic Pathology, Kyushu University (Fukuoka, Japan). All of the patients were Japanese, and the mean age of the patients with primary tumors was 66 years (35 males and 40 females). Radiation-induced sarcomas and secondary sarcomas after chemotherapy were excluded. The diagnosis of UPS was reassessed using to the 2013 World Health Organization classification of tumors [1]. Follow-up information was available in 73 tumor cases.

We evaluated the extent of necrosis and mitosis in order to be able to define the French Federation of Cancer Centers (FNCLCC) grade of each tumor [13]. The seventh edition of the American Joint Committee on Cancer (AJCC) staging system [14] was applied to every case. The Institutional Review Board at the Kyushu University approved this retrospective study (permission code 27-330).

\section{Cell Culture and Reagents}

The human UPS cell lines FPS- 1 and FU-MFH-2 were provided by the Fukushima Medical University and Fukuoka University, and cultured in RPMI-1640 and Dulbecco's modified Eagle's medium/F-12, respectively $[15,16]$. These medium preparations were supplemented with $10 \%$ fetal bovine serum (FBS) plus penicillin and streptomycin. The HSP90 inhibitor alvespimycin (17-dimethylaminoethylamino-17-demethoxygeldanamycin; 17 DMAG) and the Erk inhibitor PD0325901 were purchased from Seleck Chemicals (Houston, Tex., USA) and Santa Cruz Biotechnology (Santa Cruz, Calif., USA), respectively, and were diluted in dimethyl sulfoxide. We selected 17-DMAG because it leads to the dephosphorylation and inactivation of Akt in UPS [12].

\section{Immunohistochemistry}

Immunohistochemical (IHC) staining was performed as described [17]. Seventy-nine formalin-fixed paraffin-embedded samples were available. Antigen retrieval was performed by boiling the slides with $10 \mathrm{mM}$ sodium citrate ( $\mathrm{pH}$ 6.0) or target retrieval solution (Dako, Carpinteria, Calif., USA). The rabbit antibodies for phosphorylated (p)-STAT3 $\left(\mathrm{Tyr}^{705}\right.$ ) (Cell Signaling Technology, Danvers, Mass., USA) and SOCS3 (Abcam, Cambridge, Mass., USA) and the mouse antibody for Ki-67 (MIB-1) (Dako) were used as primary antibodies. All antibodies were diluted at 1:100 (with 5\% dry skimmed milk-TBS for SOCS3). The immune complex was detected with the EnVision Detection System (Dako).

p-STAT3 immunoreactivity was judged as positive when the proportion of tumor cells that stained more strongly than adjacent endothelial cells was $\geq 10 \%$ [5]. SOCS3 immunopositivity was scored from 0 to 4 for the percentage of positive cells and from 0 to 3 for staining intensity with reference to a previous report [18]. SOCS3 was defined as overexpressed if the overall score (percentage score $\times$ intensity score) was $>3$. The percentage of immunoreactive cells and staining intensity were evaluated in the most representative areas. The percentage of MIB-1-immunopositive cells is referred to as the MIB-1 labeling index. The MIB-1 labeling index was evaluated as described [12].

\section{Western Blotting}

We conducted Western blot analysis as described [19]. Protein was extracted from 10 frozen tumor samples paired with skeletal muscle tissue and the two UPS cell lines before and after 24-hour treatment with $300 \mathrm{nmol} / \mathrm{l}$ 17-DMAG or $100 \mathrm{nmol} / \mathrm{l}$ PD0325901. The rabbit antibodies for p-STAT3 $\left(\mathrm{Tyr}^{705}\right)$, total $(\mathrm{t})$-STAT3, pAkt $\left(\operatorname{Ser}^{473}\right)$, t-Akt, p-Erk1/2 $\left(\mathrm{Thr}^{202} / \mathrm{Tyr}^{204}\right)$, t-Erk1/2 (Cell Signaling Technology), and SOCS3 (Abcam) were used as primary antibodies. These antibodies were diluted 1:400. Anti-glyceraldehyde 3-phosphate dehydrogenase (GAPDH) (1:5,000 dilution; Santa Cruz Biotechnology) mouse monoclonal antibody was used as an internal control.

We calculated the phosphorylation scores (p-scores) using the formula reported by Setsu et al. [20] as follows: [p-protein (tumor)/t-protein (tumor)]/[p-protein (normal)/t-protein (normal)]. This formula was applied to STAT3. We compared the intensity of SOCS3 with that of GAPDH instead of $t$-protein.

\section{Migration Assay}

We performed a Boyden chamber assay to evaluate the motility of the UPS cell lines. Cells are placed in the upper chamber 
Table 1. Clinicopathological parameters and survival analysis ( $\chi 2$ or log-rank test) of 75 primary UPS patients

\begin{tabular}{|c|c|c|c|c|c|c|}
\hline Variable & & $\mathrm{n}$ & $\%$ & Analyses & OS & EFS \\
\hline \multirow[t]{2}{*}{ Sex } & Male & 30 & 40 & & 0.1429 & 0.1011 \\
\hline & Female & 45 & 60 & & & \\
\hline \multirow[t]{2}{*}{$\overline{\text { Age }}$} & $<66$ years & 37 & 49.3 & & 0.1873 & 0.1819 \\
\hline & $\geq 66$ years & 38 & 50.7 & & & \\
\hline \multirow[t]{3}{*}{ Size } & $<5 \mathrm{~cm}$ & 30 & 40 & & 0.0084 & 0.0020 \\
\hline & $\geq 5 \mathrm{~cm}$ & 44 & 58.7 & & & \\
\hline & n.a. & 1 & 1.3 & & & \\
\hline \multirow[t]{3}{*}{ Location } & Superficial & 36 & 48 & & 0.0136 & 0.0082 \\
\hline & Deep & 38 & 50.7 & & & \\
\hline & n.a. & 1 & 1.3 & & & \\
\hline \multirow[t]{2}{*}{ Metastasis } & + & 27 & 36 & & $<0.0001$ & \\
\hline & - & 48 & 64 & & & \\
\hline \multirow[t]{2}{*}{ Recurrence } & + & 15 & 20 & & 0.7347 & \\
\hline & - & 60 & 80 & & & \\
\hline \multirow[t]{3}{*}{ Necrosis } & 0, score 0 & 48 & 64 & score 0 vs. others & 0.0020 & 0.0036 \\
\hline & $<50 \%$, score 1 & 18 & 24 & score 1 vs. score 2 & 0.2386 & 0.5794 \\
\hline & $\geq 50 \%$, score 2 & 9 & 12 & score 2 vs. others & 0.0079 & 0.0478 \\
\hline \multirow[t]{3}{*}{ Mitosis } & 0-9/10 HPF, score 1 & 38 & 50.7 & score 1 vs. others & 0.0001 & 0.0004 \\
\hline & $10-19 / 10$ HPF, score 2 & 22 & 29.3 & score 2 vs. score 3 & 0.0042 & 0.2521 \\
\hline & $20 \geq / 10 \mathrm{HPF}$, score 3 & 15 & 20 & score 3 vs. others & $<0.0001$ & 0.0010 \\
\hline \multirow[t]{3}{*}{ MIB-1 LI } & $<10 \%$ & 20 & 26.7 & & 0.0569 & 0.0533 \\
\hline & $\geq 10 \%$ & 50 & 66.7 & & & \\
\hline & n.a. & 5 & 6.6 & & & \\
\hline \multirow[t]{2}{*}{ FNCLCC } & 2 & 47 & 62.7 & & $<0.0001$ & 0.0002 \\
\hline & 3 & 28 & 37.3 & & & \\
\hline \multirow[t]{3}{*}{$\overline{\mathrm{AJCC}}(\mathrm{ed} 7)$} & II & 43 & 57.3 & stage II vs. others & $<0.0001$ & $<0.0001$ \\
\hline & III & 6 & 8 & stage III vs. IV & 0.1951 & 0.0006 \\
\hline & IV & 26 & 34.7 & stage IV vs. others & 0.0001 & 0.0001 \\
\hline
\end{tabular}

Mean age: 66 years (range 17-93). LI = Labeling index; n.a. = not available; $\mathrm{n}=$ number of patients. Significant values are italicized.

separated by an 8 -mm pore size filter in serum-free media. The cells were allowed to migrate into the lower compartment, which was filled with media containing 5\% FBS. The upper chambers were treated with human recombinant interleukin (IL)-6 (25 ng/ $\mathrm{ml}$ ) (PeproTech Inc) with or without STAT3 inhibitor (S3I-201; Santa Cruz Biotechnology) (100 nM) for $48 \mathrm{~h}$.

Cells were incubated for $24 \mathrm{~h}$, and migrating cells were fixed to the lower surface of the membrane with $70 \%$ ethanol, stained with hematoxylin and eosin, and counted in 5 random fields at $\times 200$ magnification.

\section{Statistical Analysis}

Continuous variables are presented as the mean \pm SD. Correlations between the parameters were analyzed using the $\chi^{2}$ test. Survival correlations are illustrated with Kaplan-Meier curves, and survival analyses were performed using the log-rank test. In multivariate analysis, a Cox proportional hazards model was used to examine risk factors identified by univariate analysis of clinicopathological parameters and IHC results.

\section{Results}

\section{Patients and Clinicopathological Findings}

The clinicopathological and prognostic data of the 75 primary tumor patients are summarized in table 1 . Univariate analysis identified several significant factors for a poor prognosis regarding overall survival (OS): large tumor size $(\geq 50 \mathrm{~mm} ; \mathrm{p}=0.0084)$, deep location $(\mathrm{p}=$ $0.0136)$, presence of tumor metastasis $(p<0.0001)$ and necrosis $(p=0.0020)$, frequent mitosis $[\geq 10 / 10$ highpower field (HPF); $\mathrm{p}=0.0001]$, FNCLCC grade 3 ( $\mathrm{p}<0.0001)$, and high AJCC stage (III or IV; $\mathrm{p}<0.0001$ ).

Similarly, poor prognostic factors for event-free survival (EFS) were large tumor size ( $\geq 50 \mathrm{~mm} ; \mathrm{p}=0.0020)$, deep location $(\mathrm{p}=0.0082)$, presence of necrosis $(\mathrm{p}=$ $0.0036)$, frequent mitosis ( $\geq 10 / 10 \mathrm{HPF} ; \mathrm{p}=0.0004)$, FNCLCC grade $3(\mathrm{p}=0.0002)$, and high AJCC stage (III or IV; $\mathrm{p}<0.0001)$. 


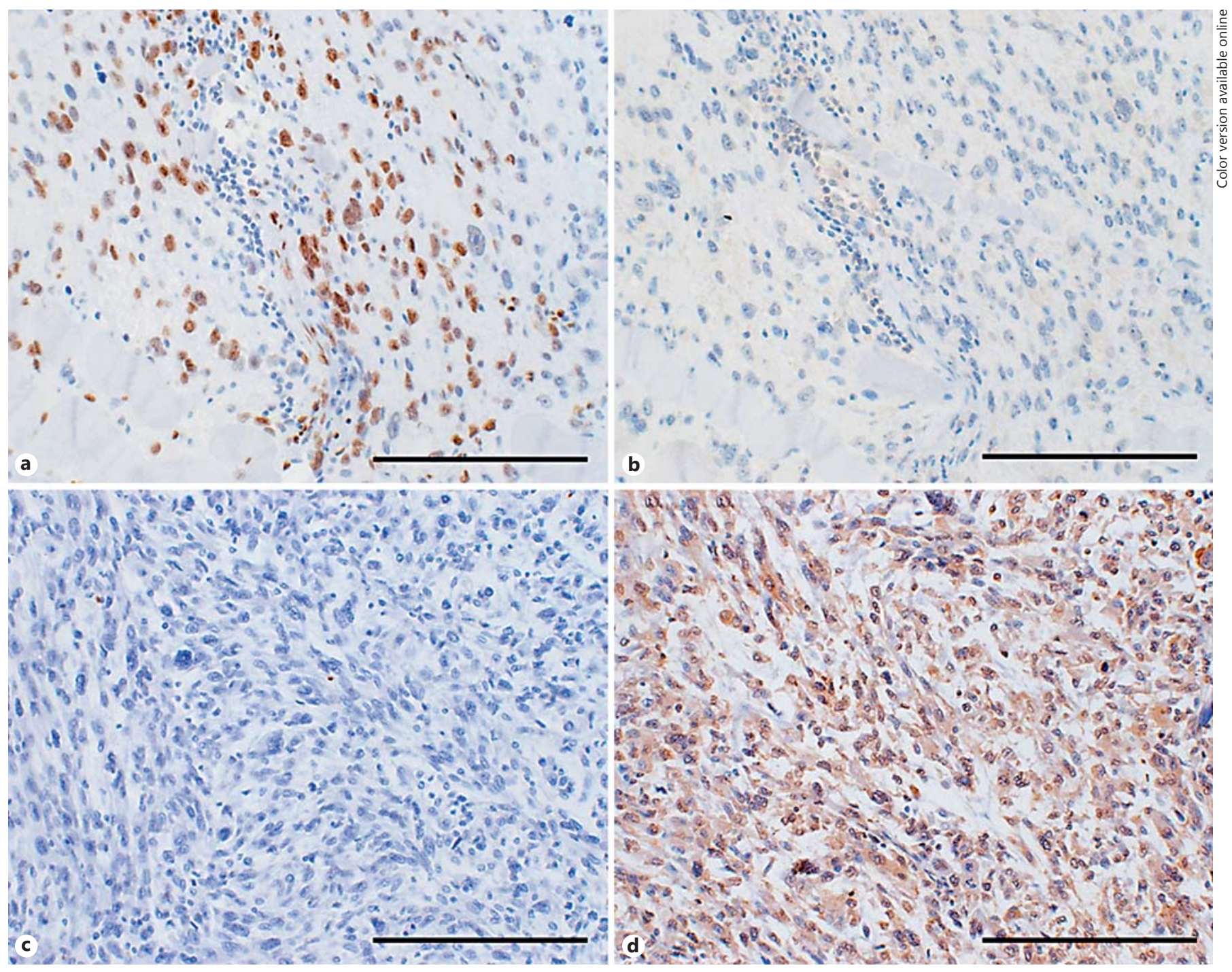

Fig. 1. IHC staining results. a This case was interpreted as positive for p-STAT3. b p-STAT3-positive UPSs were significantly negative for SOCS3 (the same case as in a). c, d p-STAT3-negative (c) and SOCS3-positive (d) cases. Nuclear and/or cytoplasmic IHC staining was judged as positive. Bar: $50 \mu \mathrm{m}$.

Multivariate analysis demonstrated that the high AJCC stage was a poor prognostic risk factor in OS ( $p<$ 0.0001; hazard ratio, 6.9).

\section{JAK-STAT Pathway IHC Results}

IHC results for p-STAT3 and SOCS3 are illustrated in figure 1 and summarized in online supplementary table S1 (see www.karger.com/doi/10.1159/000448524 for all online suppl. material). The correlation between the IHC results and the clinicopathological data are summarized in table 2. The positive ratios for p-STAT3 and SOCS3 were 59.7 and $55.8 \%$, respectively. The cases in which endothe- lial cells failed to reveal any staining were excluded from the evaluation (p-STAT3, 2 cases; SOCS3, 1 case).

The negativity for $\mathrm{p}$-STAT3 and the positivity for SOCS3 were correlated with each other $(\mathrm{p}=0.0223)$. Clinicopathologically, the positivity for p-STAT3 was correlated with low AJCC stage ( $\mathrm{p}=0.0201)$, and the positivity for SOCS3 was correlated with large tumor size ( $\mathrm{p}=$ $0.0469)$ and presence of metastasis $(p=0.0453)$. In the univariate analysis, the negativity for p-STAT3 and the positivity for SOCS3 were significant prognosticators for both poor OS ( $\mathrm{p}-\mathrm{STAT} 3, \mathrm{p}=0.0006$; SOCS3, $\mathrm{p}=0.0001$ ) and EFS ( $\mathrm{p}$-STAT3, $\mathrm{p}=0.017$; SOCS3, $\mathrm{p}=0.0125$; table 2). 


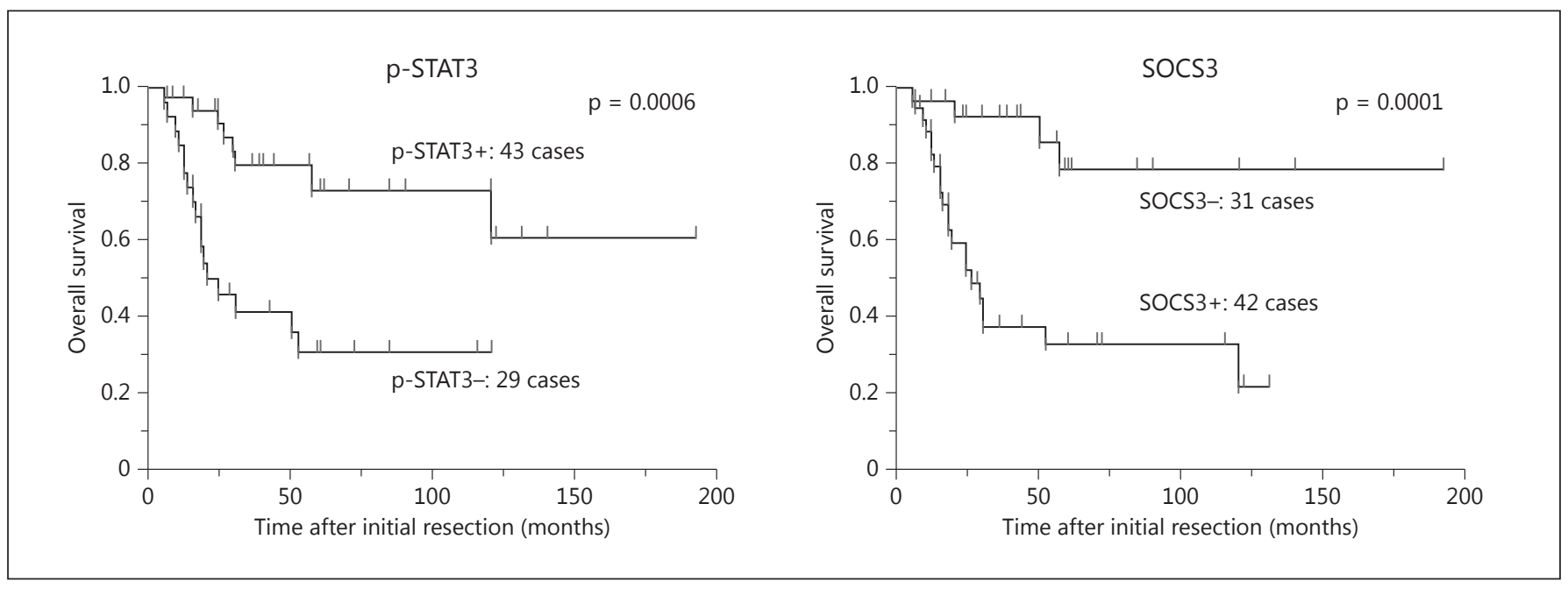

Fig. 2. Kaplan-Meier survival curves for overall survival according to the IHC results.

Table 2. Statistical analysis ( $\chi^{2}$ or log-rank test) of IHC results

\begin{tabular}{lll}
\hline Variable & \multicolumn{2}{l}{ p value } \\
\cline { 2 - 3 } & p-STAT3+ & SOCS3+ \\
\hline Sex & 0.6075 & 0.4803 \\
Age & 0.7445 & 0.3611 \\
Size & 0.2371 & 0.0469 \\
Location & 0.7763 & 0.0527 \\
Metastasis & 0.0718 & 0.0453 \\
Recurrence & 0.6792 & 0.5318 \\
Necrosis (absence vs. presence) & 0.3903 & 0.0912 \\
Mitotic activity (0-9 vs. 10/10 HPF) & 0.4003 & 0.1828 \\
MIB-1 labeling index & 0.6998 & 0.2923 \\
FNCLCC & 0.1013 & 0.0593 \\
AJCC (stage II vs. III/IV) & 0.0201 & 0.1053 \\
OS & 0.0006 & 0.0001 \\
EFS & 0.017 & 0.0125 \\
\hline
\end{tabular}

Significant values are italicized.

The Kaplan-Meier survival curves for OS according to the IHC results are illustrated in figure 2. The multivariate analysis indicated that negativity for p-STAT3 and positivity for SOCS3 were significant prognosticators for poor OS (p-STAT3, p = 0.0058; SOCS3, $\mathrm{p}=0.0012)(\mathrm{ta}-$ ble 3$)$.

\section{Western Blotting Using Frozen Samples}

The results of Western blotting are shown in figure 3 . Densitometric analysis demonstrated that p-STAT3 expression achieved p-scores $<1.0$ in all cases, indicating
Table 3. Multivariate survival analysis of IHC parameters

\begin{tabular}{lll}
\hline Parameter $^{\mathrm{a}}$ & HR $(95 \% \mathrm{CI})$ & p value \\
\hline p-STAT3 & $3.3(1.4-8.3)$ & 0.0058 \\
SOCS3 & $4.8(1.7-16.8)$ & 0.0012 \\
\hline \multicolumn{2}{c}{ CI = Confidence interval; HR = hazard ratio. } \\
\multicolumn{2}{c}{ a Adjusted by AJCC stage and tumor location. } \\
\hline
\end{tabular}

that STAT3 was phosphorylated in the tumor tissue at a significantly lower level than in the nonneoplastic tissue (mean 0.05 vs. $0.26, \mathrm{p}=0.0414$ ) (fig. 4 ). SOCS3 expression showed p-scores $>1.0$ in all cases. The level of SOCS3 expression in the tumor tissue was significantly higher in the tumor samples than in the nonneoplastic tissue (mean 1.08 vs. $0.22, \mathrm{p}=0.0102$ ) (fig. 4 ).

\section{Relationship between p-STAT3 and Other Signaling \\ Pathways}

We reported that the Akt/mTOR pathway was activated and the MAPK pathway tended to be activated in UPS [12]. Based on IHC results, the positivity for p-AKT was not significantly correlated with SOCS3 expression $(\mathrm{p}=0.1322)$, and the positivity for $\mathrm{p}$-Erk $1 / 2$ was not significantly correlated with $\mathrm{p}$-STAT3 expression $(\mathrm{p}=$ 0.5328).

The effects of 17-DMAG and PD0325901 in the two UPS cell lines are illustrated in figure 5. Human muscle tissue was used as a positive control (online suppl. fig. 1).

Pathobiology 2017;84:161-169 DOI: $10.1159 / 000448524$ 
Fig. 3. Protein expression by Western blot analysis. For STAT3, all 10 cases showed pscores $<1.0$, which suggests that STAT3 is phosphorylated to a lesser extent in tumor than nonneoplastic tissue. For SOCS3, all 10 cases showed SOCS3/GAPDH ratios $>1.0$. SOCS3 expression was higher in tumor $(\mathrm{T})$ than nonneoplastic tissue $(\mathrm{N})$.

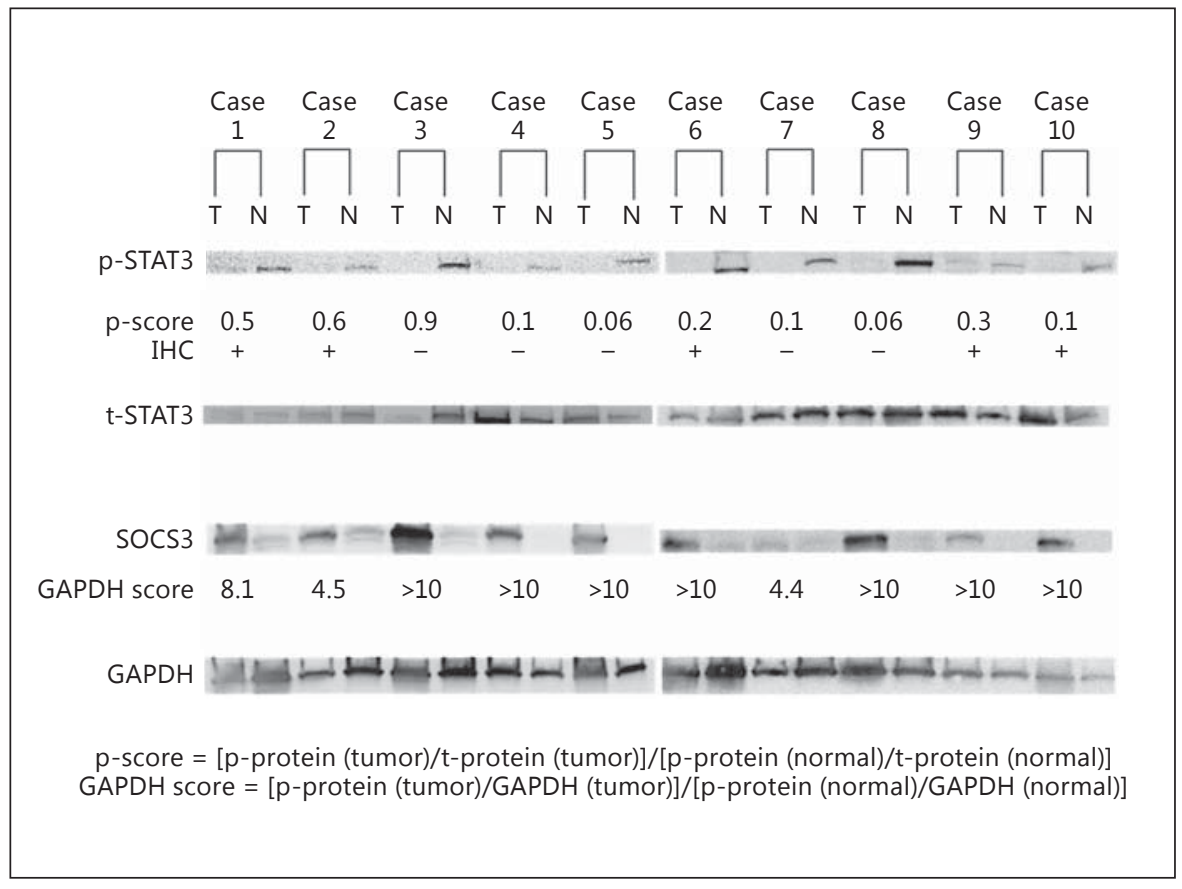

p-score $=[p$-protein (tumor)/t-protein (tumor)] $/[$ p-protein (normal)/t-protein (normal) $]$ GAPDH score $=[$ p-protein (tumor)/GAPDH (tumor)]/[p-protein (normal)/GAPDH (normal)]

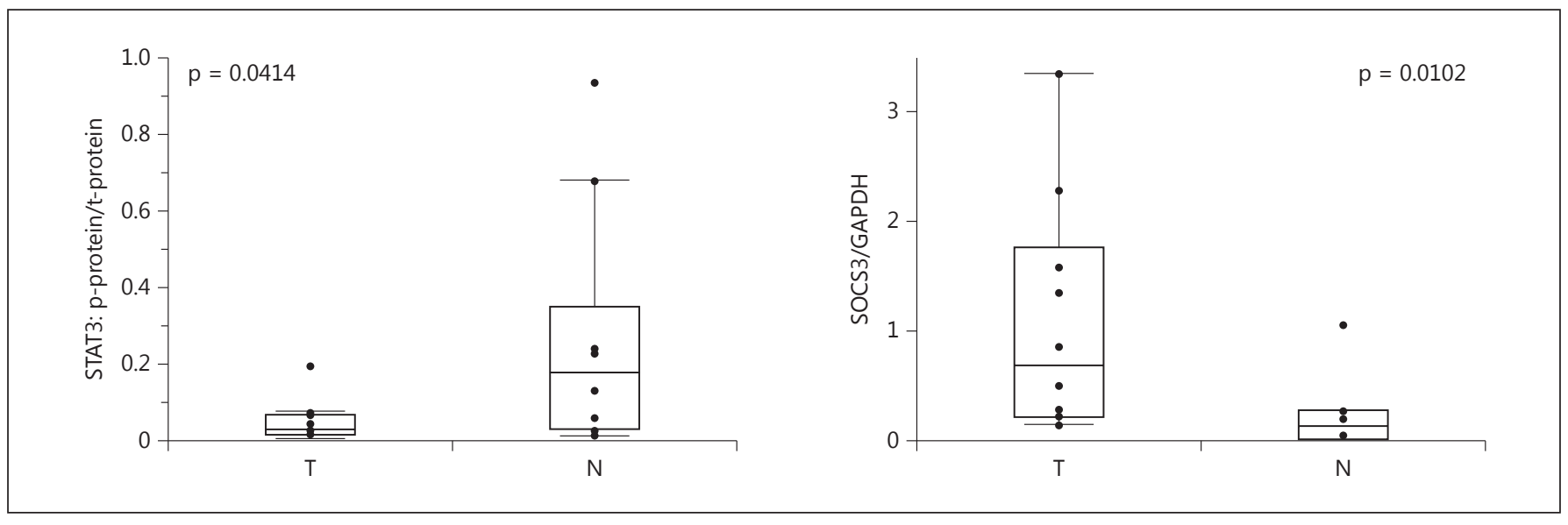

Fig. 4. Differences in protein expression between the tumor and normal tissue. STAT3 was phosphorylated in the tumor tissue at a significantly lower level than in nonneoplastic tissue $(\mathrm{p}=0.0414)$. SOCS3 expression was significantly higher in tumor $(\mathrm{T})$ than in nonneoplastic tissue $(\mathrm{N})(\mathrm{p}=0.0102)$.

The inactivation of Akt by 17-DMAG and Erk1/2 by PD0325901 induced no detectable change in the expression of p-STAT3.

\section{Mortality of Cell Lines after STAT3 Inhibition}

There was no change in the motility of the two UPS cell lines in our comparison of treated and control cells (data not shown).

\section{Discussion}

The purpose of this study was to clarify the expression of p-STAT3 in UPS. Our findings demonstrated that STAT3 dephosphorylation is an independent risk factor for a poor prognosis in UPS. Our results also suggest that SOCS3 overexpression may influence the STAT3 dephosphorylation in UPS. p-STAT3 was dephosphorylated re- 


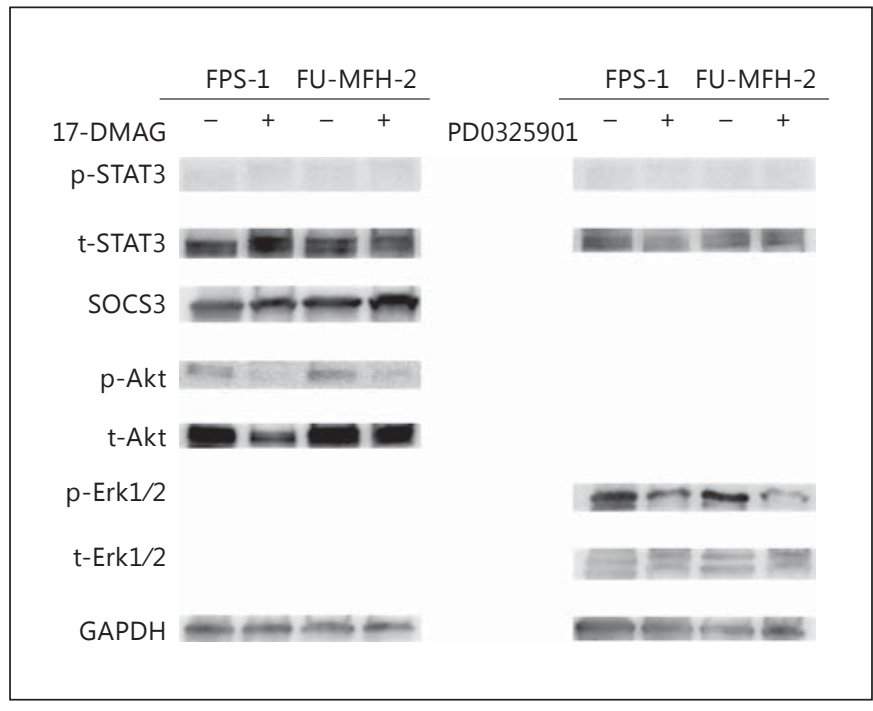

Fig. 5. Changes in protein expression in the JAK-STAT pathway. UPS cells were treated with $300 \mathrm{nmol} / \mathrm{l}$ of $17-\mathrm{DMAG}$ or $100 \mathrm{nmol} / \mathrm{l}$ of PD0325901 once $(0 \mathrm{~h})$, and cells were harvested $24 \mathrm{~h}$ later. $\mathrm{t}$ protein levels of p-STAT3 and SOCS3 were not changed by 17DMAG. Inactivation of Erk1/2 by PD0325901 induced no detectable changes in p-STAT3 expression. GAPDH was used as a loading control.

gardless of the status of the Akt/mTOR and MAPK pathways.

When cytokines bind to their receptors, conformational changes in the receptors activate JAK and then STAT. STAT factors dimerize and translocate into the nucleus to initiate the transactivation of target genes [21]. The activation of STAT3 has been described in many solid and hematological tumors [22]. STAT3 activation is correlated with a better or worse prognosis depending on the tumor type [3-6]. In either case, dysfunction of the JAK/STAT pathway seems to be involved in the development of malignant tumors.

Our Western blotting analysis showed that STAT3 was phosphorylated at a lower level in the tumor than nonneoplastic tissue. IHC results showed that STAT3 was inactivated in approximately $40 \%$ of the UPS samples, and that STAT3 inactivation was associated with a worse prognosis. However, the antitumor function of STAT3 in UPS remains to be clarified, because STAT3 phosphorylation was not correlated with any other clinicopathological parameter except for AJCC stage.

SOCS negatively regulates the JAK-STAT signaling pathway [23]. SOCS3 is an inducible negative-feedback inhibitor of cytokine signaling [2]. Suppression of SOCS3 causes the development of cancer cells in the human lung

p-STAT3 in UPS
[24]. On the other hand, SOCS3 overexpression suppresses the apoptosis pathway in prostate cancer and is associated with decreased survival in leiomyosarcoma [5, 25]. The function of SOCS3 in neoplasms thus remains controversial.

The present Western blotting analysis showed that SOCS3 expression was higher in tumor than nonneoplastic tissue. We conducted this IHC study to clarify correlations between SOCS3 expression and clinicopathological parameters. IHC revealed that positivity for SOCS3 was associated with a worse prognosis, and that it was correlated with old age and the presence of metastasis. SOCS3 was negatively correlated with p-STAT3, which suggested that SOCS3 overexpression plays an inhibitory effect on STAT3 activation in UPS.

On the basis of our analysis results of the clinical samples, we conducted an in vitro study of the JAK-STAT pathway. One of the signaling molecules that could foster STAT3 inactivation is Akt. Akt modulates cellular function in response to extracellular signals and can lead to tumor development and progression [9]. A study of osteoclasts showed that Akt activation is necessary for SOCS3 expression [10]. To analyze the correlation between the JAK-STAT pathway and p-Akt, we performed targeted inhibition of p-Akt on UPS cells using 17DMAG, one of the HSP90 inhibitors, because 17-DMAG inactivated the kinase activity of Akt [12]. HSP90 protects p-Akt from dephosphorylation, and the inhibition of Akt-HSP90 binding leads to the dephosphorylation and inactivation of Akt.

The results revealed no detectable changes in p-STAT3 and SOCS3 expression regardless of the status of p-Akt. Expression of t-STAT3 was apparently higher in the FPS1 lane with 17-DMAG than in the control FPS-1 lane, which suggests that Akt negatively regulates t-STAT3 and possibly p-STAT3. However, there was no detectable change in the SOCS3 expression, which indicates that $\mathrm{p}$ Akt has no direct influence on the STAT3 status. However, the results shown in the t-STAT3 panel may be due to our technique.

Another signaling molecule that could foster STAT3 inactivation is Erk. Erk1/2 is a member of the MAPK cascade and antagonizes the phosphorylation of STAT3 [11]. To analyze the correlation between the JAK-STAT pathway and Erk1/2, we performed targeted inhibition of pErk1/2 on UPS cells using PD0325901, a selective Erk 1/2 inhibitor. The results revealed no detectable changes in p-STAT3 expression regardless of the status of p-Erk1/2. This suggested that STAT3 inactivation was independent of the MAPK pathway in UPS.

Pathobiology 2017;84:161-169 167 
We attempted to clarify whether STAT3 inhibition can suppress the motility of UPS cell lines using a Boyden chamber assay. As in an earlier study [26], we used a STAT3 inhibitor and human recombinant IL- 6 to modify the status of p-STAT3. The results showed no change in the motility of the two cell lines. Since prior research indicated that STAT3 phosphorylation decreased cell proliferation in malignant fibrous histiocytoma cells, the role of STAT3 may differ depending on the type of cells examined [7].

We concentrated on the prognostic utility of STAT3 and SOCS3 in clinical samples, and we did not verify the status of IL-6 or its receptor in UPS. Nakanishi et al. [7] suggested that the IL-6 signaling pathway plays an important role in the proliferation, invasion, and morphology of malignant fibrous histiocytoma cells via the STAT3 pathway. Analyses of the IL-6 pathway in clinical samples would provide us an in-depth understanding of JAK-
STAT pathway activation. Despite this limitation, the results of the present study may have important clinical implications, because this is the first study to analyze the prognostic significance of the STAT3 status in UPS.

In conclusion, we elucidated the STAT3 status in UPS by IHC, Western blotting, and in vitro studies. SOCS3 may determine the activation status of this transcription factor. Although the role of the JAK-STAT pathway in UPS remains to be elucidated in more detail, the present findings provide the important clinical implication that p-STAT3 could be a useful prognostic factor for UPS.

\section{Acknowledgments}

This work was supported in part by a Grant-in-Aid for Scientific Research (B) (25293088) from the Japanese Society for the Promotion of Science (to Y.O.).

\section{References}

1 Fletcher CDM, Chibon F, Mertens F: Unclassified sarcoma; in Fletcher CDM, Bridge JA, Hogendoorn PCW, Mertens F (eds): World Health Organization Classification of $\mathrm{Tu}$ mours. Pathology and Genetics of Tumours of Soft Tissue and Bone. Lyon, IARC Press, 2013, pp 235-238.

2 White CA, Nicola NA: SOCS3: an essential physiological inhibitor of signaling by interleukin-6 and G-CSF family cytokines. JAKSTAT 2013;2:e25045.

3 Li WC, Ye SL, Sun RX, Liu YK, Tang ZY, Kim Y, Karras JG, Zhang H: Inhibition of growth and metastasis of human hepatocellular carcinoma by antisense oligonucleotide targeting signal transducer and activator of transcription 3. Clin Cancer Res 2006;12:71407148.

4 Xie TX, Huang FJ, Aldape KD, Kang SH, Liu M, Gershenwald JE, Xie K, Sawaya R, Huang S: Activation of STAT3 in human melanoma promotes brain metastasis. Cancer Res 2006; 66:3188-3196.

5 Setsu N, Kohashi K, Endo M, Yamamoto H, Tamiya S, Takahashi Y, Yamada Y, Ishii T, Matsuda S, Yokoyama R, Iwamoto Y, Oda Y: Phosphorylation of signal transducer and activator of transcription 3 in soft tissue leiomyosarcoma is associated with a better prognosis. Int J Cancer 2013;132:109-115.

6 Pectasides E, Egloff AM, Sasaki C, Kountourakis P, Burtness B, Fountzilas G, Dafni U, Zaramboukas T, Rampias T, Rimm D, Grandis J, Psyrri A: Nuclear localization of signal transducer and activator of transcription 3 in head and neck squamous cell carcinoma is associated with a better prognosis. Clin Cancer Res 2010;16:2427-2434.

7 Nakanishi H, Yoshioka K, Joyama S, Araki N, Myoui A, Ishiguro S, Ueda T, Yoshikawa $\mathrm{H}$, Itoh $\mathrm{K}$ : Interleukin-6/soluble interleukin-6 receptor signaling attenuates proliferation and invasion, and induces morphological changes of a newly established pleomorphic malignant fibrous histiocytoma cell line. Am J Pathol 2004;165:471-480.

8 Shouda T, Hiraoka K, Komiya S, Hamada T, Zenmyo M, Iwasaki H, Isayama T, Fukushima N, Nagata K, Yoshimura A: Suppression of IL-6 production and proliferation by blocking STAT3 activation in malignant soft tissue tumor cells. Cancer Lett 2006;231:176-184.

9 Fresno Vara JA, Casado E, de Castro J, Cejas P, Belda-Iniesta C, González-Barón M: PI3K/ Akt signalling pathway and cancer. Cancer Treat Rev 2004;30:193-204.

10 Ruan M, Pederson L, Bradley EW, Bamberger AM, Oursler MJ: Transforming growth factor- $\beta$ coordinately induces suppressor of cytokine signaling 3 and leukemia inhibitory factor to suppress osteoclast apoptosis. Endocrinology 2010;151:1713-1722.

11 Chung J, Uchida E, Grammer TC, Blenis J: STAT3 serine phosphorylation by ERK-dependent and -independent pathways negatively modulates its tyrosine phosphorylation. Mol Cell Biol 1997;17:6508-6516.

12 Bekki H, Kohashi K, Maekawa A, Yamada Y, Yamamoto H, Harimaya K, Hakozaki M, Nabeshima K, Iwamoto Y, Oda Y: Elevated ex- pression of HSP90 and the antitumor effect of an HSP90 inhibitor via inactivation of the Akt/mTOR pathway in undifferentiated pleomorphic sarcoma. BMC Cancer 2015;15:804.

13 Trojani M, Contesso G, Coindre JM, Rouesse J, Bui NB, de Mascarel A, Goussot JF, David M, Bonichon F, Lagarde C: Soft-tissue sarcomas of adults; study of pathological prognostic variables and definition of a histopathological grading system. Int J Cancer 1984;33: $37-42$.

14 Edge SB, Byrd DR, Compton CC (eds): AJCC Cancer Staging Manual, ed 7. St. Louis, Springer, 2010.

15 Hakozaki M, Hojo H, Sato M, Tajino T, Yamada H, Kikuchi S, Abe M: Establishment and characterization of a new cell line, FPS-1, derived from human undifferentiated pleomorphic sarcoma, overexpressing epidermal growth factor receptor and cyclooxygenase- 2 . Anticancer Res 2006;26:3393-3401.

16 Nishio J, Iwasaki $H$, Nabeshima K, Ishiguro M, Isayama T, Naito M: Establishment of a new human pleomorphic malignant fibrous histiocytoma cell line, FU-MFH-2: molecular cytogenetic characterization by multicolor fluorescence in situ hybridization and comparative genomic hybridization. J Exp Clin Cancer Res 2010;29:153.

17 Yamada Y, Kohashi K, Fushimi F, Takahashi Y, Setsu N, Endo M, Yamamoto H, Tokunaga S, Iwamoto Y, Oda Y: Activation of the AktmTOR pathway and receptor tyrosine kinase in patients with solitary fibrous tumors. Cancer 2014;120:864-876. 
18 Ying M, Li D, Yang L, Wang M, Wang N, Chen Y, He M, Wang Y: Loss of SOCS3 expression is associated with an increased risk of recurrent disease in breast carcinoma. J Cancer Res Clin Oncol 2010;136:1617-1626.

19 Mizuuchi Y, Aishima S, Ohuchida K, Shindo K, Fujino M, Hattori M, Miyazaki T, Mizumoto K, Tanaka M, Oda Y: Anterior gradient 2 downregulation in a subset of pancreatic ductal adenocarcinoma is a prognostic factor indicative of epithelial-mesenchymal transition. Lab Invest 2015;95:193-206.

20 Setsu N, Yamamoto H, Kohashi K, Endo M, Matsuda S, Yokoyama R, Nishiyama K, Iwamoto Y, Dobashi Y, Oda Y: The Akt/mammalian target of rapamycin pathway is acti- vated and associated with adverse prognosis in soft tissue leiomyosarcoma. Cancer 2012; 118:1637-1648.

$21 \mathrm{Yu} \mathrm{H}$, Jove R: The STATs of cancer - new molecular targets come of age. Nat Rev Cancer 2004;4:97-105.

22 Bar-Natan M, Nelson EA, Xiang M, Frank DA: STAT signaling in the pathogenesis and treatment of myeloid malignancies. JAKSTAT 2012;1:55-64.

23 Rakesh K, Agrawal DK: Controlling cytokine signaling by constitutive inhibitors. Biochem Pharmacol 2005;70:649-657.

24 He B, You L, Uematsu K, Zang K, Xu Z, Lee AY, Costello JF, McCormick F, Jablons DM: SOCS-3 is frequently silenced by hypermeth- ylation and suppresses cell growth in human lung cancer. Proc Natl Acad Sci USA 2003; 100:14133-14138.

25 Puhr M, Santer FR, Neuwirt H, Susani M, Nemeth JA, Hobisch A, Kenner L, Culig Z: Downregulation of suppressor of cytokine signaling-3 causes prostate cancer cell death through activation of the extrinsic and intrinsic apoptosis pathways. Cancer Res 2009;69: 7375-7384.

26 Mano Y, Aishima S, Fujita N, Tanaka Y, Kubo Y, Motomura T, Taketomi A, Shirabe K, Maehara Y, Oda Y: Tumor-associated macrophage promotes tumor progression via STAT3 signaling in hepatocellular carcinoma. Pathobiology 2013;80:146-154. 